\title{
Attitude Determination via GNSS Carrier Phase and Inertial Aiding
}

\author{
Daniel Medina ${ }^{1,2}$, Vincenzo Centrone ${ }^{3}$, Ralf Ziebold ${ }^{1}$, and Jesús García ${ }^{2}$ \\ ${ }^{1}$ Institute of Communications and Navigation, German Aerospace Center (DLR), Germany \\ ${ }^{2}$ Computer Science Engineering Department, Universidad Carlos III de Madrid, Spain \\ ${ }^{3}$ Department of Electronics and Telecommunications (DET), Politecnico di Torino, Italy
}

\begin{abstract}
Attitude Determination (AD) constitutes an important navigation component for vehicles that require orientation information, such as spacecraft or ships. Global Navigation Satellite Systems (GNSS) enable resolving the orientation of a vehicle in a precise and absolute manner, by employing a setup of multiple GNSS antennas rigidly mounted onboard the tracked vehicle. To achieve high-precision attitude estimation based on GNSS, the use of carrier phase observations becomes indispensable, with the consequent added complexity of resolving the integer ambiguities. The use of inertial aiding has been extensively exploited for $\mathrm{AD}$, since it enables tracking fast rotation variations and bridging short periods of GNSS outage. In this work, the fusion of inertial and GNSS information is exploited within the recursive Bayesian estimation framework, applying an Error State Kalman Filter (ESKF). Unlike common Kalman Filters, ESKF tracks the error or variations in the state estimate, posing meaningful advantages for $\mathrm{AD}$. On the one hand, ESKF represents attitude using a minimal state representation, in form of rotation vector, avoiding attitude constraints and singularity risks on the covariance matrix estimates. On the other hand, second-order products on the derivation of the Jacobian matrices can be neglected, since the errorstate operates always close to zero. This work details the procedure of recursively estimating the attitude based on the fusion of GNSS and inertial sensing. The GNSS attitude model is parametrized in terms of quaternion rotation, and the overall three-steps $\mathrm{AD}$ procedure (float estimation, ambiguity resolution and solution fixing) is presented. The method performance is assessed on a Monte Carlo simulation, where different noise levels, number of satellites and baseline lengths are tested. The results show that the inertial aiding, along with a constrained attitude model for the float estimation, significantly improve the performance of attitude determination compared to classical unaided baseline tracking.
\end{abstract}

Keywords - Attitude Determination, GNSS, Inertial Navigation, Error State Kalman Filter (ESKF)

\section{INTRODUCTION}

With the growing interest towards autonomous vehicles, it is of great importance to guarantee the accuracy and reliability of navigation estimates provided by Global Navigation Satellite Systems (GNSS). Other than providing the absolute localization and timing, GNSS signals can be used to compute the orientation of a platform when multiple antennas are used [1]. Attitude determination is the process of estimating the orientation of a rigid body with respect to a reference frame and it constitutes a fundamental task for the navigation of vehicles that require directional guidance. GNSS represents an appealing alternative to magnetomers, gyroscopes or other means of orientation tracking, providing a drift-less absolute attitude solution while posing a fair compromise in terms of cost, weight and precision. Employing a setup of multiple GNSS antennas rigidly mounted on a vehicle, one seeks to find the rotation which relates the local and global navigation frames. Accurate attitude estimation based on GNSS requires the use of carrier phase observations. These are characterized by a noise two orders of magnitude lower than code pseudorange observations, but they are ambiguous by an unknown number of integer ambiguities. Ambiguity Resolution (AR) is the process of resolving the unknown cycle ambiguities of the carrier phase as integers [2]. The sole purpose of AR is to use the integer ambiguity constraints as a means of significantly improving on the navigation solution. The GNSS attitude model is generally expressed as a Least-squares (LS) adjustment, for which closed-form solution is not known. Thus, its minimization is resolved applying a three-steps decomposition. The first step float estimation constitutes a LS procedure where the integer constraint on the ambiguities is dropped. Then, the AR estimates the integer ambiguities based on the float solution. This stage is commonly solved applying the Least-squares AMBiguity Decorrelation Adjustment (LAMBDA) method [3-5]. Finally, solution fixing consists on improving the attitude solution based on the estimated integer ambiguities. 
Traditionally, the GNSS compass model relates the double difference observations to the baseline vectors relating the antennas, expressed in the global frame. The use of this model in combination with the standard LAMBDA method has been often applied for AD [6-9]. However, classical LAMBDA is not designed for AD, due to the nonlinear constraints related to the prior knowledge of the distance and relative orientation between the antennas [10]. Aiming to improve LAMBDA for GNSS carrier phase-based AD, Teunissen and Giorgi introduced the Constrained-LAMBDA (C-LAMBDA) and the Multivariate Constrained-LAMBDA (MC-LAMBDA) methods. First, C-LAMBDA modifies the original LAMBDA method introducing the information on the baseline length as a constraint, exploited during the process of AR and solution fixing [11-13]. Although C-LAMBDA is shown to provide significant improvement against classical LAMBDA, additional information on the relative orientation between antennas is not included. In MC-LAMBDA, the constraints on separation and orientation between antennas is incorporated, supporting the float solution and assisting on the integer ambiguity search. To do so, the attitude matrix becomes the state estimate, increasing the complexity due to the constraints on orthogonality and determinant equal to one [14-18]. This work proposes the formulation of the GNSS attitude model based on the quaternion parametrization of the rotation. As MC-LAMBDA, the proposed model fully exploits the knowledge on the distance and relative orientation between antennas and its use is valid also for rank-deficient scenario, e.g. when the antennas are aligned in the same direction or in a coplanar configuration. A clear asset is the use of a minimal state parametrization compared to the estimate of the rotation matrix. More importantly, the use of the quaternion is convenient for multisensor recursive estimation, since the equations for the quaternion kinematics are linear.

Inertial sensors have been often used in combination with GNSS, given their immunity against radio threats and their high sensitivity to track fast motions [19-22]. Thus, the fusion of inertial and satellite information benefits from the short term performance of inertial sensors and the absolute orientation provided by GNSS. Such sensor fusion is generally realized within the recursive Bayesian estimation framework, particularly applying the nonlinear versions of the Kalman Filter (KF): the Extended Kalman Filter (EKF) and the Unscented Kalman Filter (UKF). Nonetheless, KF-based attitude determination poses certain challenges. First, the nonlinear constraints of the rotation are complicated to incorporate to the filter design. Second, if the parametrization of the attitude is not minimal, the estimation of the covariance matrix might incur numerical problems and singularity risks. In this work, an Error State Kalman Filter (ESKF) is applied to circumvent the aforementioned difficulties and singularities for AD problems. While the ESKF has been discussed for the integration of inertial data with attitude estimation derived from star trackers within the aerospace research community [23-25], its adoption to the GNSS carrier phase-based AD remains unexplored. The fusion of inertial and GNSS using ESKF is shown to enable fast and robust AR, since the latter can be more precisely initialized with the high-rate output of the inertial sensor, as well as tracking the uncertainty of the system without the risk of incurring in potential numerical problems.

This work presents a method for AD based on the fusion of inertial and GNSS information. The proposed approach comprises three phases: I) float estimation is realized using an ESKF, which integrates inertial data and GNSS information to provide estimates for the quaternion, gyroscope biases and carrier phase ambiguities. The GNSS attitude model is expressed using quaternion parametrization for the rotation, incorporating the prior knowledge on the inter-antenna separation and relative orientation [26]. II) AR is performed using a classical LAMBDA method. A major advantage of applying the ESKF consists on having a rather small search space for the integer ambiguities. This is due to the float estimation constituting a multivariate constrained attitude problem and the inertial integration avoiding errors in the system kinematics. III) The orientation and gyroscope biases are reconstructed conditioned on the integer ambiguity solution, providing a reliable and accurate estimation.

The performance of the proposed attitude determination solver has been assessed in two kind of simulation scenarios. First, the performance of the proposed quaternion-based GNSS attitude model is assessed and compared against classical unconstrained-baseline tracking. Thus, a Monte Carlo experiment is realized for a static scenario, over different baseline lengths, number of satellites and magnitude of code observation noises. This experimentation is realized in a memoryless or snapshot manner, to differ the performance of the quaternion GNSS attitude model from the benefits related to inertial integration and the recursive estimation. Then, the performance of the proposed GNSS/IMU attitude determination is addressed for a dynamical scenario. The experimentation consists of a Monte Carlo experiment, where the IMU and GNSS observations are simulated for a multi-GNSS multi-frequency scenario (GPS L1, L2 and Galileo E1, E5a). The performance characterization is realized in terms of estimated accuracy and the ratio of successful ambiguity fixing.

The structure of the paper is as follows. Section II briefly discusses on quaternion attitude parametrization. In Section III, the different GNSS attitude models are discussed. Then, Section IV introduces the overall fusion architecture. Finally, Section V and VI correspond to the experimentation, discussion and outlook. 


\section{QUATERNION PARAMETRIZATION FOR THE ATTITUDE PROBLEM}

Attitude determination is the process of finding the relative orientation between two orthogonal frames. In $\mathbb{R}^{3}$, the rotation group $\mathcal{S O}(3)$ denotes the group of rotations under the operation of composition [27]. Rotations are linear operations preserving vector length and the relative vector orientation. Thus, the rotation group $\mathcal{S O}(3)$ can be defined as

$$
\mathcal{S O}(3):=\left\{r: \mathbb{R}^{3} \rightarrow \mathbb{R}^{3} / \forall \boldsymbol{v}, \boldsymbol{w} \in \mathbb{R}^{3},\|r(\boldsymbol{v})\|=\|\boldsymbol{v}\|, r(\boldsymbol{v}) \times r(\boldsymbol{w})=r(\boldsymbol{v} \times \boldsymbol{w})\right\}
$$

The rotation operation can be represented in multiple forms, although the most well known correspond to Euler angles, rotation matrix and quaternion. Euler angles describe orientation as three consecutive elemental rotations. Although they are conceptually easy to understand, they pose a singularity problem, commonly referred to as "gimbal lock", when a degree of freedom is loss due a parallel configuration of two axes. Rotation matrices are characterized as orthonormal matrices with determination 1. Rotation matrices represents attitude without incurring in any singularity. However, rotation matrices result in difficult renormalization and computational inefficiency (rotation matrices have 9 elements to describe a 3D attitude). Finally, the quaternion is a four dimensional hyper-complex number, introduced by Hamilton in 1843 [28], which is often used to represent the orientation of a rigid body in a 3D space, under the unit-norm constraint. Although attitude quaternion might not be intuitive and difficult to be visualized, it has been widely adopted, especially in robotics and computer graphics [29,30]. In this work, the quaternion parametrization has been chosen for two practical reasons: the kinematic model of the prediction equations are linear and the quaternion rotation is free from singularities (e.g., the gimbal-lock is eluded).

This paper adopts the Hamilton convention, scalar part first and local-to-global rotation (body to Earth-Centered Earth-Fixed ECEF). For more details on the different quaternion conventions and a comprehensive explanation on quaternion algebra, the reader is referred to $[27,31,32]$. Thus, the quaternion is defined as:

$$
\boldsymbol{q} \equiv\left[\begin{array}{c}
q_{0} \\
q_{u}
\end{array}\right]
$$

with

$$
\begin{aligned}
q_{0} & =\cos (\theta / 2) \\
\boldsymbol{q}_{\boldsymbol{u}} & =\left[\begin{array}{lll}
q_{1} & q_{2} & q_{3}
\end{array}\right]^{\top}=\boldsymbol{e} \sin (\theta / 2)
\end{aligned}
$$

where $\boldsymbol{e}$ is the unit Euler axis and $\theta$ is the rotation angle. In Eq. (3), the quaternion relation to the rotation vector is appreciated. This will be of fundamental importance for the later description of the Error-State Kalman Filter formulation. The most general motion of a rigid body with respect to a fixed point is a rotation by an angle $\theta$ about an axis specified by the unit vector $\boldsymbol{e}$ [24]. Thus, given a rotation vector $\boldsymbol{\theta}$

$$
\boldsymbol{\theta} \equiv \theta \boldsymbol{e}
$$

a quaternion $\boldsymbol{q}\{\boldsymbol{\theta}\}$ can be derived as

$$
\boldsymbol{q}\{\boldsymbol{\theta}\}=\left[\begin{array}{c}
\cos \left(\frac{\|\boldsymbol{\theta}\|^{2}}{2}\right) \\
\frac{\boldsymbol{\theta}}{\|\boldsymbol{\theta}\|^{2}} \sin \left(\frac{\|\boldsymbol{\theta}\|^{2}}{2}\right)
\end{array}\right]
$$

A quaternion only describes a proper rotation under the unit-norm constraint of its components:

$$
\|\boldsymbol{q}\|^{2} \equiv q_{0}^{2}+\left\|\boldsymbol{q}_{\boldsymbol{u}}\right\|^{2}=1
$$

Quaternions obeying this constraint can be said to belong to the unit-quaternion group, denoted as $\mathbb{H}^{*}$. The transformation quaternion to rotation matrix $\boldsymbol{R}$ is given by the following homogeneous quadratic function:

$$
\boldsymbol{R}\{\boldsymbol{q}\}=\left(q_{0}^{2}-\boldsymbol{q}_{\boldsymbol{u}}^{\top} \boldsymbol{q}_{\boldsymbol{u}}\right) \boldsymbol{I}_{3}+2 \boldsymbol{q}_{\boldsymbol{u}} \boldsymbol{q}_{\boldsymbol{u}}^{\top}+2 q_{0}\left[\boldsymbol{q}_{\boldsymbol{u}} \times\right]
$$

where $\boldsymbol{I}_{3} \in \mathbb{R}^{3}$ is the identity matrix and the skew operator $[\boldsymbol{v} \times]$ defines the cross-product matrix

$$
[\boldsymbol{v} \times]=\left[\begin{array}{ccc}
0 & -v_{z} & v_{y} \\
v_{z} & 0 & -v_{x} \\
-v_{y} & v_{z} & 0
\end{array}\right]
$$


which is a skew-symmetric matrix, i.e., $[\boldsymbol{v} \times]=-[\boldsymbol{v} \times]$, equivalent to the matrix form of the cross product $[\boldsymbol{v} \times] \boldsymbol{w}=$ $\boldsymbol{v} \times \boldsymbol{w}$.

The quaternion product ${ }^{1} \circ$ is non-commutative, associative and distributive over the sum:

$$
\boldsymbol{p} \circ \boldsymbol{q}=\left[\begin{array}{c}
p_{0} q_{0}-\boldsymbol{p}_{\boldsymbol{u}}^{\top} \boldsymbol{q}_{\boldsymbol{u}} \\
p_{0} \boldsymbol{q}_{\boldsymbol{u}}+q_{0} \boldsymbol{p}_{\boldsymbol{u}}+\boldsymbol{p}_{\boldsymbol{u}} \times \boldsymbol{q}_{\boldsymbol{u}}
\end{array}\right]
$$

The composition of quaternions is bilinear and can be expressed as two matrix products:

$$
\boldsymbol{q}_{1} \circ \boldsymbol{q}_{\mathbf{2}}=\left[\boldsymbol{q}_{\mathbf{1}}\right]_{L} \boldsymbol{q}_{\mathbf{2}} \quad \boldsymbol{q}_{\mathbf{1}} \circ \boldsymbol{q}_{\mathbf{2}}=\left[\boldsymbol{q}_{\mathbf{2}}\right]_{R} \boldsymbol{q}_{\mathbf{1}}
$$

with $[\boldsymbol{q}]_{L}$ and $[\boldsymbol{q}]_{R}$ are the left and right quaternion product matrices, respectively. These product matrices are given by:

$$
[\boldsymbol{q}]_{L}=q_{0} \boldsymbol{I}_{4}+\left[\begin{array}{cc}
0 & -\boldsymbol{q}_{\boldsymbol{u}}^{\top} \\
\boldsymbol{q}_{\boldsymbol{u}} & {\left[\boldsymbol{q}_{\boldsymbol{u}} \times\right]}
\end{array}\right], \quad[\boldsymbol{q}]_{R}=q_{0} \boldsymbol{I}_{4}+\left[\begin{array}{cc}
0 & -\boldsymbol{q}_{\boldsymbol{u}}^{\top} \\
\boldsymbol{q}_{\boldsymbol{u}} & -\left[\boldsymbol{q}_{\boldsymbol{u}} \times\right]
\end{array}\right]
$$

The rotation operator over a vector $r(\boldsymbol{v})$ using quaternion parametrization is expressed as:

$$
r(\boldsymbol{v})=\boldsymbol{R}\{\boldsymbol{q}\} \boldsymbol{v}=\boldsymbol{q} \circ \boldsymbol{v} \circ \boldsymbol{q}^{*}
$$

where $\boldsymbol{q}^{*}$ is the inverse quaternion operation

$$
\boldsymbol{q}^{*}=\left[\begin{array}{c}
q_{0} \\
-\boldsymbol{q}_{\boldsymbol{u}}
\end{array}\right]
$$

The quaternion kinematics equation is given by

$$
\dot{\boldsymbol{q}} \equiv \frac{d}{d t} \boldsymbol{q}=\frac{1}{2} \boldsymbol{q} \circ\left[\begin{array}{c}
0 \\
\boldsymbol{\omega}
\end{array}\right]
$$

where $\boldsymbol{\omega}$ is the three-component angular rate vector, observed at the local frame.

\section{GNSS ATTITUDE MODELS}

Let us consider $n+1$ GNSS satellites tracked on a single frequency simultaneously at $N+1$ antennas installed on a vehicle. At a particular time, the phase and code observations at receiver $j$ of the tracked satellite $i$ is expressed as follows:

$$
\begin{gathered}
\rho_{j}^{i}=\left\|\boldsymbol{p}^{i}-\boldsymbol{p}_{j}\right\|^{2}+I^{i}+T^{i}+c\left(d t_{j}-d t^{i}\right)+\varepsilon_{j}^{i} \\
\Phi_{j}^{i}=\left\|\boldsymbol{p}^{i}-\boldsymbol{p}_{j}\right\|^{2}-I^{i}+T^{i}+c\left(d t_{j}-d t^{i}\right)+\lambda N_{j}^{i}+\epsilon_{j}^{i}
\end{gathered}
$$

where

$\rho_{j}^{i}, \Phi_{j}^{i}$ are the code and phase observations $[\mathrm{m}]$

$\boldsymbol{p}^{i}, \boldsymbol{p}_{j}$ are the positions of the $\mathrm{i}^{\text {th }}$ satellite and the $\mathrm{j}^{\text {th }}$ GNSS antenna respectively,

$I^{i}$ is the ionospheric error $[\mathrm{m}]$,

$T^{i}$ is the tropospheric error $[\mathrm{m}]$,

$c$ is the speed of light $[299792458 \mathrm{~m} / \mathrm{s}]$

$d t_{j}, d t^{i}$ are the satellite and receiver clock offsets [s],

$\lambda$ is the carrier phase wavelength $[\mathrm{m}]$,

$N^{i}$ is unknown number of cycles between the receiver and the satellite,

\footnotetext{
${ }^{1}$ The quaternion product is generally denoted as $\otimes$. However, this work reserves $\otimes$ for the representation of the Kronecker product.
} 
$\varepsilon^{i}, \epsilon^{i}$ are the remaining unmodelled errors -multipath effects, instrumental delays, phase biases, etc.- for the code and phase observations respectively.

Among the $N+1$ antennas on the vehicle, one is chosen as master (from here denoted with the subscript $m$ ). Single-differencing the observations, e.g., subtracting the observations received at the master antenna from the other antennas, removes the atmospheric and satellite-related errors. Then, the process of double-differencing, or removing the single-difference observations with respect to a pivot or reference satellite (hereafter referred with the superscript $r$ ), eliminates the clock offsets of the receivers on board of the tracked vehicle. Fig. 1 illustrates the aforementioned process and the notations.

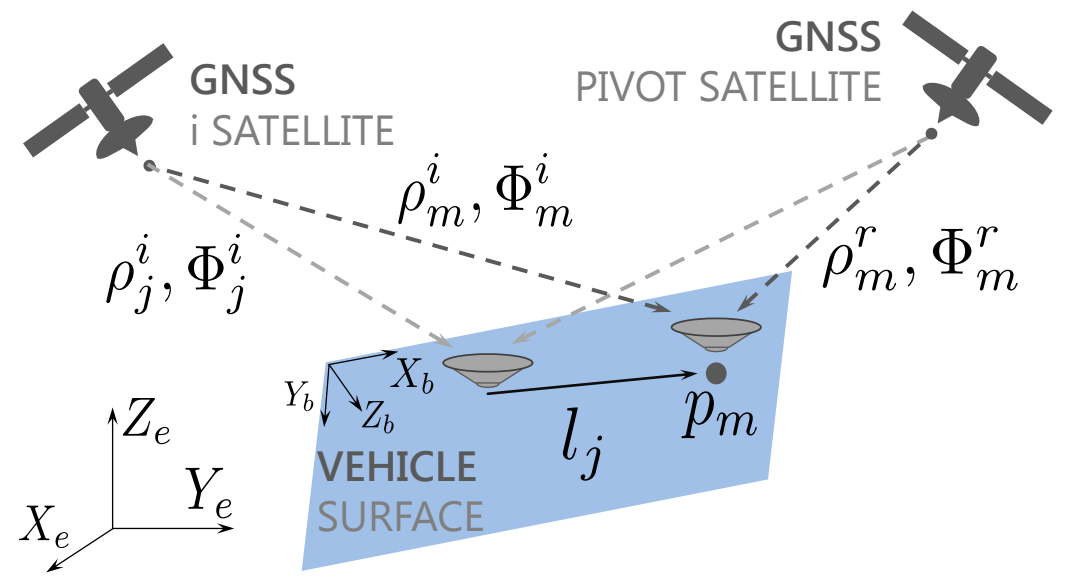

Figure 1: Diagram for the collection of GNSS data over different GNSS antennas onboard a vehicle. The baseline connecting the $j$ antenna with the master antenna $m$ expressed in the local coordinate frame of the vehicle is denoted as $\boldsymbol{l}_{\boldsymbol{j}}$. The global coordinate frame (ECEF frame) is depicted on the bottom left with the axes $X_{e} Y_{e} Z_{e}$.

The linearized functional model of the double-difference code and carrier phase measurements $D D \rho_{j}^{i}$ and $D D \Phi_{j}^{i}$ can be expressed as follows

$$
\begin{gathered}
D D \rho_{j}^{i}=\rho_{j}^{i}-\rho_{m}^{i}-\left(\rho_{j}^{r}-\rho_{m}^{r}\right) \\
=-\left(\boldsymbol{u}^{i}-\boldsymbol{u}^{r}\right)^{\top} \boldsymbol{b}_{j}+\varepsilon_{j, b}^{i, r} \\
D D \Phi_{j}^{i}=\Phi_{j}^{i}-\Phi_{m}^{i}-\left(\Phi_{j}^{r}-\Phi_{m}^{r}\right) \\
=-\left(\boldsymbol{u}^{i}-\boldsymbol{u}^{r}\right)^{\top} \boldsymbol{b}_{j}+\lambda a_{j}^{i}+\epsilon_{j, m}^{i, r}
\end{gathered}
$$

where $\boldsymbol{u}^{i}$ is satellite-to-antenna unit line-of-sight vector (due to the relatively small inter-antenna separation, the line-of-sight vectors can be considered the same among the $N+1$ antennas) and $a_{j}^{i}$ is the double difference ambiguity between $j$ and master antenna. The baseline vector between two antennas, expressed in the global frame, is denoted by $\boldsymbol{b}_{j}=\boldsymbol{p}_{j}-\boldsymbol{p}_{m}$.

The system of double-difference phase and code observations can be expressed as

$$
E(\boldsymbol{y})=\boldsymbol{A} \boldsymbol{a}+\boldsymbol{B} \boldsymbol{b}, \quad D(\boldsymbol{y})=\boldsymbol{Q}_{y y}
$$

where $E(\cdot)$ and $D(\cdot)$ are, respectively, the expectation and dispersion operators. The vector of observables $\boldsymbol{y}$ are defined as

$$
\boldsymbol{y}=\left[\boldsymbol{D} \boldsymbol{D} \boldsymbol{\Phi}_{1}^{\top}, \cdots, \boldsymbol{D} \boldsymbol{D} \boldsymbol{\Phi}_{N}^{\top}, \boldsymbol{D} \boldsymbol{D} \boldsymbol{\rho}_{1}^{\top}, \cdots, \boldsymbol{D} \boldsymbol{D} \boldsymbol{\rho}_{N}^{\top}\right]^{\top}
$$

where $\boldsymbol{y} \in \mathbb{R}^{2 n N}$ and $\boldsymbol{D} \boldsymbol{D} \boldsymbol{\Phi}_{j}, \boldsymbol{D} \boldsymbol{D} \boldsymbol{\rho}_{j} \in \mathbb{R}^{n}$ is the vector of carrier and code double differences for each of the $N$ baselines. The vector of double difference phase ambiguities $\boldsymbol{a} \in \mathbb{Z}^{n N}$ stacks the ambiguities across the baselines. Matriz $\boldsymbol{A}$ relates the observations to the vector of ambiguities as

$$
\boldsymbol{A}=\left[\begin{array}{l}
\lambda \boldsymbol{I}_{n N} \\
\boldsymbol{O}_{n M}
\end{array}\right]
$$


where $\boldsymbol{O}_{n M}$ is the square null matrix of size $n M$. The variance-covariance matrix of the observations $\boldsymbol{Q}_{y y}$ is defined as

$$
\boldsymbol{Q}_{y y}=\left[\begin{array}{cc}
\boldsymbol{P}_{N} \otimes \boldsymbol{D} \boldsymbol{Q}_{\Phi} \boldsymbol{D}^{\top} & \mathbf{0} \\
\mathbf{0} & \boldsymbol{P}_{N} \otimes \boldsymbol{D} \boldsymbol{Q}_{\rho} \boldsymbol{D}^{\top}
\end{array}\right]
$$

To account for the noise cross-correlation introduced due to double-differencing the observations, matrices $\boldsymbol{P}_{N}$ and $\boldsymbol{D}$ are defined as follows:

$$
\boldsymbol{P}_{N}=\frac{1}{2}\left(\boldsymbol{I}_{N}+\boldsymbol{I}_{N} \boldsymbol{I}_{N}^{\top}\right), \boldsymbol{D}=\left[\boldsymbol{I}_{n},-\mathbf{1}_{n}\right]
$$

where $\mathbf{1}_{n}$ is the ones vector of size $n$. The diagonal matrices of the expected uncertainty of each of the $n+1$ phase and code observations, $\boldsymbol{Q}_{\Phi}$ and $\boldsymbol{Q}_{\rho}$ respectively, are:

$$
\boldsymbol{Q}_{\Phi}=\operatorname{diag}\left(\sigma_{\Phi}^{1^{2}}, \ldots, \sigma_{\Phi}^{n+1^{2}}\right), \boldsymbol{Q}_{\rho}=\operatorname{diag}\left(\sigma_{\rho}^{1^{2}}, \ldots, \sigma_{\rho}^{n+1^{2}}\right)
$$

This work considers noise stochastic modelling based on satellite elevation el:

$$
\sigma_{\Phi, i}^{2}=a^{2}+\left(\frac{b}{\sin \left(e l_{i}\right)}\right)^{2}, \sigma_{\rho, i}^{2}=100^{2} \cdot\left(a^{2}+\left(\frac{b}{\sin \left(e l_{i}\right)}\right)^{2}\right)
$$

where $a=2 m m, b=2 m m$, as suggested in [33]. Finally, $\boldsymbol{b}$ and $\boldsymbol{B}$ represent the remaining state estimate and its associated design matrix, whose definition is discussed next.

Although the GNSS attitude problem is generally solved applying a LS adjustment, the underlying model can be expressed in different ways. Thus, the classical LAMBDA approach estimates the baseline vectors between the antennas, without incorporating the a priori knowledge on baseline length and relative orientation, as:

$$
\left\{\boldsymbol{a}_{L}, \boldsymbol{b}_{L}\right\}=\arg \min _{\boldsymbol{a} \in \mathbb{Z}^{n N}, \boldsymbol{b} \in \mathbb{R}^{3 N}}\|\boldsymbol{y}-\boldsymbol{A} \boldsymbol{a}-\boldsymbol{G} \boldsymbol{b}\|_{Q_{y y}}^{2}
$$

where $\boldsymbol{G} \in \mathbb{R}^{3 \times n N}$ is the geometry matrix of the double-difference line-of-sight vectors. Then, C-LAMBDA performs a similar adjustment, enhanced by the addition of the the constraint over the baseline length to the search-space $[11-13]$ as

$$
\left\{\boldsymbol{a}_{C}, \boldsymbol{b}_{C}\right\}=\arg \min _{\boldsymbol{a} \in \mathbb{Z}^{n N}, \boldsymbol{b} \in \mathbb{R}^{3 N},\left\|\boldsymbol{b}_{j}\right\|^{2}=\left\|\boldsymbol{l}_{j}\right\|^{2}}\|\boldsymbol{y}-\boldsymbol{A} \boldsymbol{a}-\boldsymbol{G} \boldsymbol{b}\|_{Q_{y y}}^{2}
$$

Besides not incorporating the information on the relative orientation between the antennas, classical LAMBDA and C-LAMBDA pose the disadvantage of not directly tracking the attitude. Therefore, an additional step for the AD using the estimated baseline vectors is required. Finally, MC-LAMBDA estimates the rotation matrix to relate the set of baselines in the local frame $\boldsymbol{F}=\left[\boldsymbol{l}_{1}, \ldots, \boldsymbol{l}_{N}\right]$ to the global frame. Hence, the prior knowledge on the configuration of the antennas is fully exploited [14-18]. Besides, MC-LAMBDA extends the formulation to account for the rank $q$ of matrix $\boldsymbol{F}$, dealing with rank-deficient attitude problems. Thus, the minimization problem becomes:

$$
\left\{\boldsymbol{a}_{M}, \boldsymbol{R}_{M}\right\}=\arg \min _{\boldsymbol{a} \in \mathbb{Z}^{n N}, \boldsymbol{R} \in \mathbb{O}^{3 \times q}}\|\boldsymbol{y}-\boldsymbol{A} \boldsymbol{a}-\boldsymbol{G} \boldsymbol{R} \boldsymbol{F}\|_{Q_{y y}}^{2}
$$

MC-LAMBDA has been shown to be the most promising GNSS attitude model so far [14-16]. Nonetheless, tracking the rotation matrix implies the estimation of nine parameters (for configurations of non-coplanar antennas) which pose only three degrees of freedom due to the orthonormality constraint.

This work proposes a GNSS attitude model based on the quaternion parametrization of the rotation. Then, the functional model for double-difference observations becomes

$$
\begin{aligned}
D D \rho_{j}^{i} & =-\left(\boldsymbol{u}^{i}-\boldsymbol{u}^{r}\right)^{\top}\left(\boldsymbol{q} \circ \boldsymbol{l}_{j} \circ \boldsymbol{q}^{*}\right)+\varepsilon_{j, m}^{i, r} \\
D D \Phi_{j}^{i} & =-\left(\boldsymbol{u}^{i}-\boldsymbol{u}^{r}\right)^{\top}\left(\boldsymbol{q} \circ \boldsymbol{l}_{j} \circ \boldsymbol{q}^{*}\right)^{+} \lambda a_{j}^{i}+\epsilon_{j, m}^{i, r}
\end{aligned}
$$

The estimation of the attitude based on quaternion rotation is performed also based on a LS adjustment as

$$
\{\boldsymbol{a}, \boldsymbol{q}\}=\arg \min _{a \in \mathbb{Z}^{n N}, \boldsymbol{q} \in \mathbb{H}^{*}}\|\boldsymbol{y}-\boldsymbol{A} \boldsymbol{a}-h(\boldsymbol{q})\|_{\boldsymbol{Q}_{y y}}^{2}
$$


The minimization posed in Eq. (31) constitutes an overdetermined system of nonlinear equations, whose resolution can be solved applying Lagrange multipliers or a Gauss-Newton method. Thus, the design matrix $B$ defines the Jacobian of the nonlinear model $h(\boldsymbol{q})$ as follows

$$
\boldsymbol{B}=\left[\mathbf{1}_{2} \otimes\left[\begin{array}{c}
\boldsymbol{G} \boldsymbol{J}_{q}\left(\boldsymbol{l}_{1}\right) \\
\vdots \\
\boldsymbol{G J}_{q}\left(\boldsymbol{l}_{N}\right)
\end{array}\right]\right]
$$

where $\boldsymbol{J}_{q}\left(\boldsymbol{l}_{j}\right)$ is the Jacobian matrix of the baseline $\boldsymbol{l}_{j}$ with respect to the quaternion rotation, whose derivation is provided in Appendix A. The procedure to resolve this minimization problem is detailed on the following section.

\section{SENSOR FUSION ARCHITECTURE FOR ATTITUDE DETERMINATION}

Similarly to the minimization problems in Eqs. (26 27, 28), a closed-form solution for Eq. (31) does not exist, due to the integer nature of the ambiguities. Minimization problems of such type are generally referred to as integer least-squares estimation, for which [3] derived a three-step solution based on the decomposition of the problem. This work adopts the same decomposition

$$
\begin{aligned}
\min _{\boldsymbol{a} \in \mathbb{Z}^{n N}, \boldsymbol{q} \in \mathbb{H}^{*}}\|\boldsymbol{y}-\boldsymbol{A} \boldsymbol{a}-h(\boldsymbol{q})\|_{\boldsymbol{Q}_{y y}}^{2}= & \min _{\hat{\boldsymbol{a}} \in \mathbb{R}^{n N}, \hat{\boldsymbol{q}} \in \mathbb{H}^{*}}\|\boldsymbol{y}-\boldsymbol{A} \hat{\boldsymbol{a}}-\boldsymbol{B} \hat{\boldsymbol{q}}\|_{\boldsymbol{Q}_{y y}}^{2} \\
& +\min _{a \in \mathbb{Z}^{n N}}\|\hat{\boldsymbol{a}}-\boldsymbol{a}\|_{\boldsymbol{Q}_{\hat{a} \hat{a}}}^{2}+\min _{\boldsymbol{q} \in \mathbb{H}^{*}}\|\check{\boldsymbol{q}}(\boldsymbol{a})-\boldsymbol{q}\|_{\boldsymbol{Q}_{\check{q}(a) \check{q}(a)}^{2}}^{2}
\end{aligned}
$$

where the first term $\|\boldsymbol{e}\|_{Q_{y y}}^{2}$ corresponds to a LS where the integer constraints on the ambiguities are dropped. The output of this estimation is referred to as float solution and its realized applying a KF (in the recursive case) or a Gauss-Newton procedure (in the snapshot or memoryless case). The second term corresponds to the AR process, estimated applying a Integer Least Squares [1,3,34]. Finally, the computed integer ambiguities are used to improve the solution for the orientation $\boldsymbol{q}$. Such estimate is realized, once again in a least-squares sense, to obtain the fixed solution. Fig. 2 illustrates the workflow of the estimation previously described. This work exploits the fusion from GNSS observations with inertial aiding, and thus a recursive KF is applied for the estimation of the float solution.

The workflow applied in this work is based on the same decomposition, and it is illustrated in Fig. 2. The first stage is the computation of a least-squares where the integer constraint of the ambiguities is dropped. The output of this estimation is referred to as float solution. Then, the estimated float ambiguities and the corresponding variancecovariance matrix are used for the AR process. Finally, the computed integer ambiguities are used to improve the solution for the orientation $\boldsymbol{q}$. Such estimate is realized, once again in a least-squares sense, to obtain the fixed solution. Next, it is detailed the estimation for three steps aforementioned.

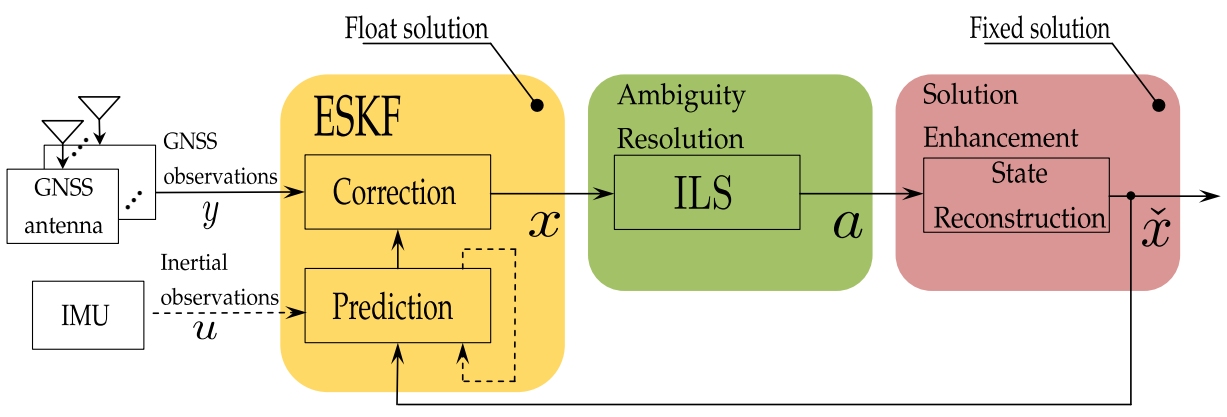

Figure 2: Workflow of the sensor fusion architecture for the inertial-aided GNSS carrier phase-based attitude determination problem.

\section{ESKF - Float Estimation}

The use of quaternion as the attitude state presents certain difficulties in the application of the classical Kalman filter equations, due to the lack of independence of the four quaternion elements, related by the unit norm constraint 
$[24,31,35,36]$. In this work, we focus on the Error-State Kalman Filter (ESKF), also found under the name of Indirect Kalman Filter [23, 25]. [32, 37] reviews the application of ESKF for AD and highlights its advantages as: i) the error-state uses a minimal parametrization, in form of rotation vector, for the rotation. Thus, redundancy issues related to the unit-norm constraint is avoided, preventing also possible singularity risks during the covariance matrix estimation. ii) Since the error-state operates close to the origin, second-order products can be neglected. Thus, the Jacobian computation becomes easier to pose and estimate.

In his work [32], Solà provides a comprehensive explanation of the ESKF operation. The ESKF applies the concept of total-, nominal- and error-state values, with the total-state expressed with the composition of the nominal- and the error- states. The rationale behind the ESKF is to consider the nominal-state as the regular state estimate of a KF (whose prediction and correction models are applied using the original nonlinear functions) and the errorstate as a small-signal gathering the noises and perturbances of the system (linearly integrable and compatible with linear KF). During the prediction step, driven by the high-update rate of the IMU, the nominal state $\hat{\boldsymbol{x}}$ integrates the inertial data disregarding possible noise terms - therefore accumulating errors. Such errors are represented within the error-state $\boldsymbol{\delta} \boldsymbol{x}$, which incorporates the system noises and perturbations. Since the error-state is conformed by small magnitudes, its evolution function can be expressed as a linear dynamic system. Thus, during the prediction step, the ESKF predicts a Gaussian estimate of the error-state. Upon the reception of GNSS data and the performance of the correction step, the filter estimates the errors observable. This correction provides a posterior Gaussian estimate of the error-state which is then injected to the nominal-state and then reset to zero.

In this work, the state estimate is conformed by the following total and error states:

$$
\hat{\boldsymbol{x}}=\left[\hat{\boldsymbol{q}}^{\top}, \hat{\boldsymbol{b}}_{\boldsymbol{\omega}}^{\top}, \hat{\boldsymbol{a}}^{\top}\right]^{\top}, \quad \boldsymbol{\delta} \boldsymbol{x}=\left[\boldsymbol{\delta} \boldsymbol{\theta}^{\top}, \boldsymbol{\delta} \boldsymbol{b}_{\boldsymbol{\omega}}^{\top}, \boldsymbol{\delta} \boldsymbol{a}^{\top}\right]^{\top},
$$

and the composition operation $\oplus$ defines the total state as

$$
\boldsymbol{x}=\hat{\boldsymbol{x}} \oplus \boldsymbol{\delta} \boldsymbol{x}=\left[\begin{array}{c}
\hat{\boldsymbol{q}} \circ \boldsymbol{\delta} \boldsymbol{q}\{\boldsymbol{\delta} \boldsymbol{\theta}\} \\
\hat{\boldsymbol{b}}_{\boldsymbol{\omega}}+\boldsymbol{\delta} \boldsymbol{b}_{\boldsymbol{\omega}} \\
\hat{\boldsymbol{a}}+\boldsymbol{\delta} \boldsymbol{a}
\end{array}\right]
$$

where $\boldsymbol{b}_{\boldsymbol{\omega}}$ represents the gyroscope biases. The total quaternion is defined by the rotation of the estimated and the error state quaternions $\boldsymbol{q}$ and $\boldsymbol{\delta} \boldsymbol{q}$. The error quaternion is obtained from the rotation vector $\boldsymbol{\delta} \boldsymbol{\theta}$. Since the rotation associated with the error quaternion can be considered small, the small angle approximation can be used to define the attitude error as a rotation vector $\boldsymbol{\delta} \boldsymbol{\theta}$, as seen in Eqs. (2 -4). The uncertainty of the system is gathered in the

covariance matrix $\boldsymbol{P} \in \mathbb{R}^{3+3+n N}$, representing the uncertainty of the attitude estimation (of three dimensions, as of the rotation vector $\boldsymbol{\delta} \boldsymbol{\theta}$ ), the gyroscope bias and the carrier phase ambiguities. The prediction and correction steps are described next.

\section{Prediction Step}

During the prediction step, the total state kinematics corresponds to the dynamical model without any noises or perturbations, while the covariance is updated based on the simpler kinematics of the error-state as follows:

$$
\boldsymbol{x}_{k}=f\left(\boldsymbol{x}_{k-1}, \boldsymbol{u}_{k}\right), \quad \boldsymbol{P}_{k}=\boldsymbol{F}_{x_{k}} \boldsymbol{P}_{k-1} \boldsymbol{F}_{x_{k}}^{\top}+\boldsymbol{F}_{q_{k}} \boldsymbol{Q}_{u} \boldsymbol{F}_{q_{k}}^{\top}
$$

where the subscript $k$ references the update time and $\boldsymbol{u}$ is the input for the prediction step, in this case the measured angular rate $\boldsymbol{\omega} . F_{x}$ and $F_{q}$ are the Jacobian matrices of the error-state kinematics with respect to the state and the control noise $\boldsymbol{Q}_{u}$ respectively. Thus, the total state kinematics are as next

$$
\begin{aligned}
\hat{\boldsymbol{q}}_{k} & =\hat{\boldsymbol{q}}_{k-1} \circ \boldsymbol{\delta} \boldsymbol{q}\left\{\left(\boldsymbol{\omega}_{\boldsymbol{k}}-\hat{\boldsymbol{b}}_{\boldsymbol{\omega}, \boldsymbol{k}-\mathbf{1}}\right) \Delta t\right\} \\
\hat{\boldsymbol{b}}_{\boldsymbol{\omega}, \boldsymbol{k}} & =\hat{\boldsymbol{b}}_{\boldsymbol{\omega}, k-1} \\
\hat{\boldsymbol{a}}_{k} & =\hat{\boldsymbol{a}}_{k-1}
\end{aligned}
$$

where the quaternion integrating the angular rate $\boldsymbol{\delta} \boldsymbol{q}$ is defined using Eq. (5). The dynamics for the error-state are as follows

$$
\begin{aligned}
\boldsymbol{\delta} \boldsymbol{\theta}_{k} & =[\boldsymbol{R}\{\boldsymbol{\delta} \boldsymbol{q}\}]^{\top} \boldsymbol{\delta} \boldsymbol{\theta}_{k-1}-\boldsymbol{\delta} \boldsymbol{b}_{\boldsymbol{\omega}, \boldsymbol{k}-1} \Delta t+\boldsymbol{\eta}_{\boldsymbol{\omega}} \Delta t \\
\boldsymbol{\delta} \boldsymbol{b}_{\boldsymbol{\omega}, k} & =\boldsymbol{\delta} \boldsymbol{b}_{\boldsymbol{\omega}, \boldsymbol{k}-\mathbf{1}}+\boldsymbol{\eta}_{\boldsymbol{b}_{\boldsymbol{\omega}} \Delta t} \\
\boldsymbol{\delta} \boldsymbol{a}_{k} & =\boldsymbol{\delta} \boldsymbol{a}_{k-1}+\boldsymbol{\eta}_{\boldsymbol{a}} \Delta t
\end{aligned}
$$


where the rotation matrix relation to the inertial integration $\boldsymbol{R}\{\boldsymbol{\delta} \boldsymbol{q}\}$ is obtained applying Eq. (7). The control noises $\boldsymbol{\eta}_{\boldsymbol{b}_{\boldsymbol{\omega}}}, \boldsymbol{\eta}_{\boldsymbol{\omega}}, \boldsymbol{\eta}_{\boldsymbol{a}}$, corresponding to the gyroscope bias, the angular rate observations and the phase ambiguities are follow a normal distribution of zero mean and variance $\sigma_{b_{\omega}}, \sigma_{\omega}, \sigma_{\omega}$ respectively. Thus, $\boldsymbol{Q}_{u}$ gets defined as

$$
\boldsymbol{Q}_{\boldsymbol{u}}=\operatorname{diag}\left(\sigma_{b_{\omega}}^{2} \boldsymbol{I}_{3}, \sigma_{\omega}^{2} \boldsymbol{I}_{3}, \sigma_{a}^{2} \boldsymbol{I}_{n N}\right) .
$$

From Eq. 38, the derivation of the Jacobian matrices $\boldsymbol{F}_{x}$ and $\boldsymbol{F}_{q}$ becomes straightforward

$$
\boldsymbol{F}_{x}=\left[\begin{array}{ccc}
{[\boldsymbol{R}\{\boldsymbol{\delta} \boldsymbol{q}\}]^{\top}} & -\boldsymbol{I} \Delta t & \boldsymbol{O}_{3, n N} \\
\mathbf{0}_{3} & \boldsymbol{I}_{3} & \mathbf{0}_{3, n N} \\
\mathbf{0}_{n N, 3} & \mathbf{0}_{n N, 3} & \boldsymbol{I}_{n N}
\end{array}\right], \quad \boldsymbol{F}_{q}=\left[\begin{array}{ccc}
\Delta t \boldsymbol{I}_{3} & \mathbf{0}_{3} & \mathbf{0}_{3, n N} \\
\mathbf{0}_{3} & \Delta t \boldsymbol{I}_{3} & \mathbf{0}_{3, n N} \\
\mathbf{0}_{n N, 3} & \mathbf{0}_{n N, 3} & \boldsymbol{I}_{n N}
\end{array}\right]
$$

\section{Correction Step}

Integrating the GNSS double-difference observations within the ESKF highly resembles the optimization problem introduced in Eq. (31), with the addition of the gyroscope biases and the special handling of the error-state rotation vector. Considering that the observations relates to the total state as

$$
\boldsymbol{y}=h(\boldsymbol{x})+\boldsymbol{\eta}
$$

where $h(\cdot)$ is the nonlinear observation model of the system state and $\eta \sim \mathcal{N}\left(0, \boldsymbol{Q}_{y y}\right)$. Since ESKF estimates on the covariance matrix relates to the error-state, the innovation vector of the KF correction constitutes the error-state:

$$
\begin{aligned}
\boldsymbol{K} & =\boldsymbol{P} \boldsymbol{H}^{\top}\left(\boldsymbol{H} \boldsymbol{P} \boldsymbol{H}^{\top}+\boldsymbol{Q}_{y y}\right)^{-1} \\
\boldsymbol{\delta} \boldsymbol{x} & =\boldsymbol{K}(\boldsymbol{y}-h(\boldsymbol{x})) \\
\boldsymbol{x} & =\hat{\boldsymbol{x}} \oplus \boldsymbol{\delta} \boldsymbol{x} \\
\boldsymbol{P} & =\left(\boldsymbol{I}_{\mathbf{3}+\mathbf{3}+\boldsymbol{n} \boldsymbol{N}}-\boldsymbol{K} \boldsymbol{H}\right) \boldsymbol{P}
\end{aligned}
$$

thus requiring the Jacobian matrix $\boldsymbol{H}$ to be defined with respect to the error-state. Applying the chain rule to the total-state, the design matrix $\boldsymbol{H}$ is obtained as

$$
\left.\boldsymbol{H} \equiv \frac{\partial h}{\partial \boldsymbol{\delta} \boldsymbol{x}}\right|_{\boldsymbol{x}}=\left.\left.\frac{\partial h}{\partial \boldsymbol{x}_{\text {true }}}\right|_{\boldsymbol{x}} \frac{\partial \boldsymbol{x}_{\text {true }}}{\partial \boldsymbol{\delta} \boldsymbol{x}}\right|_{\boldsymbol{x}}=\boldsymbol{H}_{x} \boldsymbol{H}_{\boldsymbol{\delta} \boldsymbol{x}}
$$

where $\boldsymbol{H}_{\boldsymbol{x}}$ is the conventional Jacobian with respect to the nominal-state:

$$
\boldsymbol{H}_{\boldsymbol{x}}=\left[\begin{array}{lll}
\boldsymbol{B} & \mathbf{0}_{2 n N, 3} & \boldsymbol{A}
\end{array}\right]
$$

and $\boldsymbol{H}_{\boldsymbol{\delta} \boldsymbol{x}}$ is defined as follows:

$$
\boldsymbol{H}_{\boldsymbol{\delta} \boldsymbol{x}}=\left[\begin{array}{ccc}
\boldsymbol{H}_{\boldsymbol{\delta} \boldsymbol{\theta}} & & \\
& \boldsymbol{I}_{3} & \\
& & \boldsymbol{I}_{n N}
\end{array}\right], \quad \boldsymbol{H}_{\boldsymbol{\delta} \boldsymbol{\theta}}=\frac{1}{2}[\boldsymbol{q}]_{L}\left[\begin{array}{ccc}
0 & 0 & 0 \\
1 & 0 & 0 \\
0 & 1 & 0 \\
0 & 0 & 1
\end{array}\right]
$$

with $\boldsymbol{B}$ and $\boldsymbol{A}$ defined in Eqs. (32), (21) respectively, and $[\boldsymbol{q}]_{L}$ is the left quaternion product matrix from Eq. (11). Once the error-state is estimated, it is injected to the total-state using the composition operation Eq. (35) and reset back to zero.

\section{Ambiguity Resolution}

Following the estimation of the float solution, AR is the process of determining the optimal set of integer ambiguities minimizing the second term in Eq. (31). Such minimization problem defines an ILS whose ellipsoidal search space lays in a multidimensional space, centered at the estimated float ambiguities. ILS is typically resolved applying the LAMBDA method. For GNSS attitude problems, C- and MC- LAMBDA propose the joint minimization of the ILS and the solution fixing procedure. Since the problem of solution fixing is affected by the code observations, its 
associated weighting matrix results much larger than the ILS one. Thus, C- and MC- LAMBDA estimates the set of integer ambiguities which minimizes the baseline length and multivariate constraints respectively.

In this work, the ILS minimization (second term in Eq. (33)) is solved separately of the solution fixing process (third term in Eq. (33)). Hence, this work solves the ILS problem applying the classical LAMBDA approach. The reasoning for such decision is that, since the integration of IMU data significantly improves the float solution estimation, the ILS ellipsoidal search space becomes reduced and finding the optimal set of integer ambiguities uncomplicated.

\section{State Reconstruction}

After the AR, the estimate of the remaining state parameters (quaternion rotation and gyroscope biases) can be enhanced upon the ambiguities integer constraint. Thus, estimated integer ambiguities behave as observations, whose influence in the state estimate is determined by the difference with the float ambiguities and the associated covariance matrices as

$$
\begin{aligned}
{\left[\begin{array}{c}
\boldsymbol{q} \\
\boldsymbol{b}_{\omega}
\end{array}\right] } & =\left[\begin{array}{c}
\hat{\boldsymbol{q}} \\
\hat{\boldsymbol{b}}_{\omega}
\end{array}\right] \ominus \check{\boldsymbol{\delta} \boldsymbol{x}}(\boldsymbol{a}), \\
\boldsymbol{\delta} \check{\boldsymbol{x}}(\boldsymbol{a}) & =\left[\begin{array}{c}
\check{\boldsymbol{\theta}}_{(\boldsymbol{a})} \\
\check{\boldsymbol{b}}_{\omega}(\boldsymbol{a})
\end{array}\right]=P_{\hat{\boldsymbol{b}} \hat{\boldsymbol{b}}} \boldsymbol{P}_{\hat{\boldsymbol{a}} \hat{\boldsymbol{a}}}(\boldsymbol{a}-\hat{\boldsymbol{a}})
\end{aligned}
$$

where $\boldsymbol{\delta} \check{\boldsymbol{x}}(\boldsymbol{a})$ refers to the error state -or innovation vector- conditioned on the estimated integer ambiguities. Similarly to the correction of the ESKF, after the injection of the error state in the nominal state, it is reset back to zero. Finally, the decomposition $\ominus$ is defined as follows

$$
\begin{aligned}
\boldsymbol{q} & =\hat{\boldsymbol{q}} \circ \boldsymbol{q}\{\check{\boldsymbol{\theta}}(\boldsymbol{a})\}^{*} \\
\boldsymbol{b}_{\omega} & =\hat{\boldsymbol{b}}_{\omega}-\check{\boldsymbol{b}}_{\omega}(\boldsymbol{a})
\end{aligned}
$$

\section{SIMULATION RESULTS}

The testing of the proposed methodology comprises two parts. First, a GNSS static scenario is simulated, with a varying number of satellites in view, baseline lengths and precision of the code observations. This allows for the characterization of the quaternion-based GNSS attitude model against the standard unconstrained-baseline GNSS attitude model, in terms of success rate (i.e., ratio of correctly ambiguity fixing) and accuracy of attitude determination. Secondly, the performance of the proposed multisensor recursive attitude determination method is addressed for a synthetic dynamical scenario. The experimentation consists of a Monte Carlo experiment, where the inertial and satellite measurements are simulated for a dynamical scenario, using GNSS frequencies L1, L2 E1 and E5a. The performance characterization is realized in terms of estimated accuracy and the ratio of successful ambiguity fixing.

\section{Quaternion-based GNSS Attitude Model Performance}

As discussed during Section 3, there are different GNSS attitude models, based on how the double-difference observations relate to the orientation. This work proposes the use of quaternions for the parametrization of the attitude, this incorporating the knowledge on the separation and relative orientation between the antennas. The LS adjustment presented in Eq. (31) constitutes a constrained nonlinear system of equations, whose solution can be estimated in different ways. For a profound discussion on methods to solve this kind of attitude problems, the reader is referred to [35]. In this work, a Gauss-Newton method is used for its solution.

The performance characterization is realized on a Monte Carlo simulation, where the GNSS observations are simulated according to the position of the satellites as illustrated in Fig. 3, across three antennas. The antennas are assumed to be placed in a non-coplanar configuration, and different baseline lengths are tested. The noise on the observations follow a Gaussian distribution with a fixed variance of three $\mathrm{mm}$ for the phase observations, while for the code observations different noise variances are tested. An overview on the simulation setup is shown is Table 1. The performance of the quaternion-based GNSS attitude model is compared to the classical unconstrained LAMBDA, where the estimate consists of the baselines in the global reference frame. 


\begin{tabular}{ll}
\hline $\begin{array}{l}\text { UTC time } \\
\text { Location }\end{array}$ & $\begin{array}{l}2017 / 05 / 15 \text { 09:30:00 } \\
\text { Koblenz, Germany } \\
\left(50^{\circ} 21^{\prime} 56^{\prime \prime} N, 7^{\circ} 35^{\prime} 55 E\right)\end{array}$ \\
\hline Frequency & L1 \\
Number of satellites & Corresponding PDOP \\
$5 / 6 / 7 / 8 / 9 / 10$ & $6.4 / 2.4 / 2.0 / 1.9 / 1.8 / 1.7$ \\
\hline Code noise $\sigma_{R}[\mathrm{~cm}]$ & $30-15-5$ \\
Phase noise $\sigma_{\Phi}[\mathrm{mm}]$ & 3 \\
Baseline length $[\mathrm{m}]$ & $0.5-2-10-50$ \\
Samples simulated & $10^{4}$ \\
\hline
\end{tabular}

Table 1: The different simulated measurement scenarios.

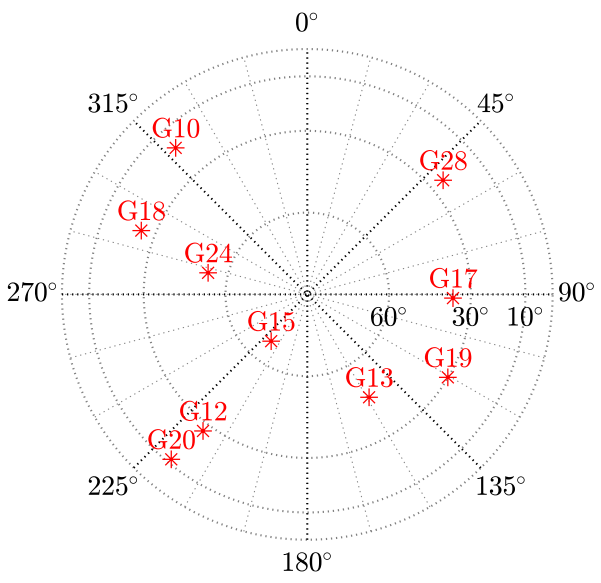

Figure 3: Sky plot of the tracked satellites for the simulations.

Table 2: Single frequency, single-epoch, percentages of successful ambiguity fixing for baseline and quaternion tracking for attitude determination.

\begin{tabular}{|c|c|c|c|c|c|c|c|c|c|c|c|c|}
\hline \multirow{2}{*}{$\begin{array}{l}\text { Baseline length }[\mathrm{m}] \\
\text { Code noise } \sigma_{R}[\mathrm{~cm}]\end{array}$} & \multicolumn{3}{|c|}{0.5} & \multicolumn{3}{|c|}{2} & \multicolumn{3}{|c|}{10} & \multicolumn{3}{|c|}{50} \\
\hline & 30 & 15 & 5 & 30 & 15 & 5 & 30 & 15 & 5 & 30 & 15 & 5 \\
\hline \multirow[t]{2}{*}{ Method } & \multicolumn{12}{|c|}{ Baseline - Tracking } \\
\hline & \multicolumn{12}{|c|}{ Quaternion - Tracking } \\
\hline \multicolumn{13}{|l|}{$n$ satellites } \\
\hline \multirow[t]{2}{*}{5} & 0.60 & 1.50 & 14.30 & 0.70 & 0.90 & 13.50 & 0.40 & 1.00 & 12.90 & 0.10 & 0.20 & 47.50 \\
\hline & 1.97 & 6.55 & 60.10 & 2.24 & 16.82 & 75.55 & 5.12 & 28.20 & 78.90 & 8.60 & 31.46 & 84.90 \\
\hline \multirow[t]{2}{*}{6} & 0.00 & 2.10 & 45.70 & 0.00 & 1.90 & 45.70 & 0.40 & 1.80 & 46.90 & 0.10 & 0.20 & 47.50 \\
\hline & 1.22 & 13.31 & 77.58 & 7.98 & 36.14 & 91.80 & 25.15 & 66.20 & 95.20 & 48.80 & 79.20 & 96.00 \\
\hline \multirow[t]{2}{*}{7} & 10.70 & 40.90 & 95.70 & 10.60 & 41.50 & 94.90 & 1.10 & 13.10 & 80.40 & 1.70 & 10.40 & 77.70 \\
\hline & 3.18 & 20.78 & 91.03 & 16.97 & 54.92 & 98.20 & 46.00 & 89.10 & 99.30 & 83.30 & 96.30 & 98.50 \\
\hline \multirow[t]{2}{*}{8} & 38.70 & 76.60 & 99.80 & 10.60 & 41.50 & 94.90 & 10.70 & 43.90 & 94.70 & 12.30 & 41.50 & 95.00 \\
\hline & 5.66 & 31.62 & 94.39 & 25.54 & 65.90 & 99.90 & 67.70 & 96.20 & 99.80 & 96.80 & 99.50 & 99.90 \\
\hline \multirow[t]{2}{*}{9} & 38.70 & 76.60 & 99.80 & 40.90 & 76.40 & 100 & 37.60 & 76.20 & 99.80 & 38.70 & 75.90 & 99.80 \\
\hline & 8.23 & 35.68 & 96.99 & 30.59 & 74.00 & 100 & 76.80 & 98.90 & 100 & 99.20 & 100 & 100 \\
\hline \multirow[t]{2}{*}{10} & 61.00 & 90.40 & 99.70 & 61.10 & 91.20 & 100 & 61.30 & 90.90 & 100 & 59.50 & 92.80 & 100 \\
\hline & 9.28 & 42.31 & 98.10 & 32.32 & 84.80 & 100 & 83.00 & 99.50 & 100 & 99.70 & 100 & 100 \\
\hline
\end{tabular}

The performance characterization is summarized in Table 2, illustrating the success rate for ambiguity resolution (the percentage of experiments where the correct set of integer ambiguities was found). Experiments for which only a set of satellites is used ( $n$ satellites $<10$ ), the discarded satellites are randomly selected in every Monte Carlo iteration. The first major difference between classical baseline and quaternion tracking is that the performance of the latter depends on the baseline length. Unlike baseline tracking, where the system of equations is linear, quaternion tracking requires solving a nonlinear problem whose resolution depends both on the baseline length and the initialization of the search space. In this experiment, the initial attitude is derived from the estimation of the three antennas using a simple code-based Single Point Positioning method [1]. Thus, when the baseline length is small and the code noise is elevated, the performance of quaternion tracking rapidly decays. A better attitude initialization could by realized by deriving the attitude from the float estimation of the baseline tracking, since the atmospheric-related errors would be removed. Besides the problem related to a poor attitude initialization in small baselines, the quaternion-based attitude model certainly overperforms classical baseline tracking. Fig. 4 illustrates the performance comparison, for 
different number of satellites and a variance for the code observations of $30 \mathrm{~cm}$, which can be considered a relatively challenging scenario. One can see that, for a high number of satellites, regular unconstrained baseline tracking success rate is always below $60 \%$. The constraints incorporated for quaternion tracking significantly enhances the performance, especially for large inter-antenna separation.
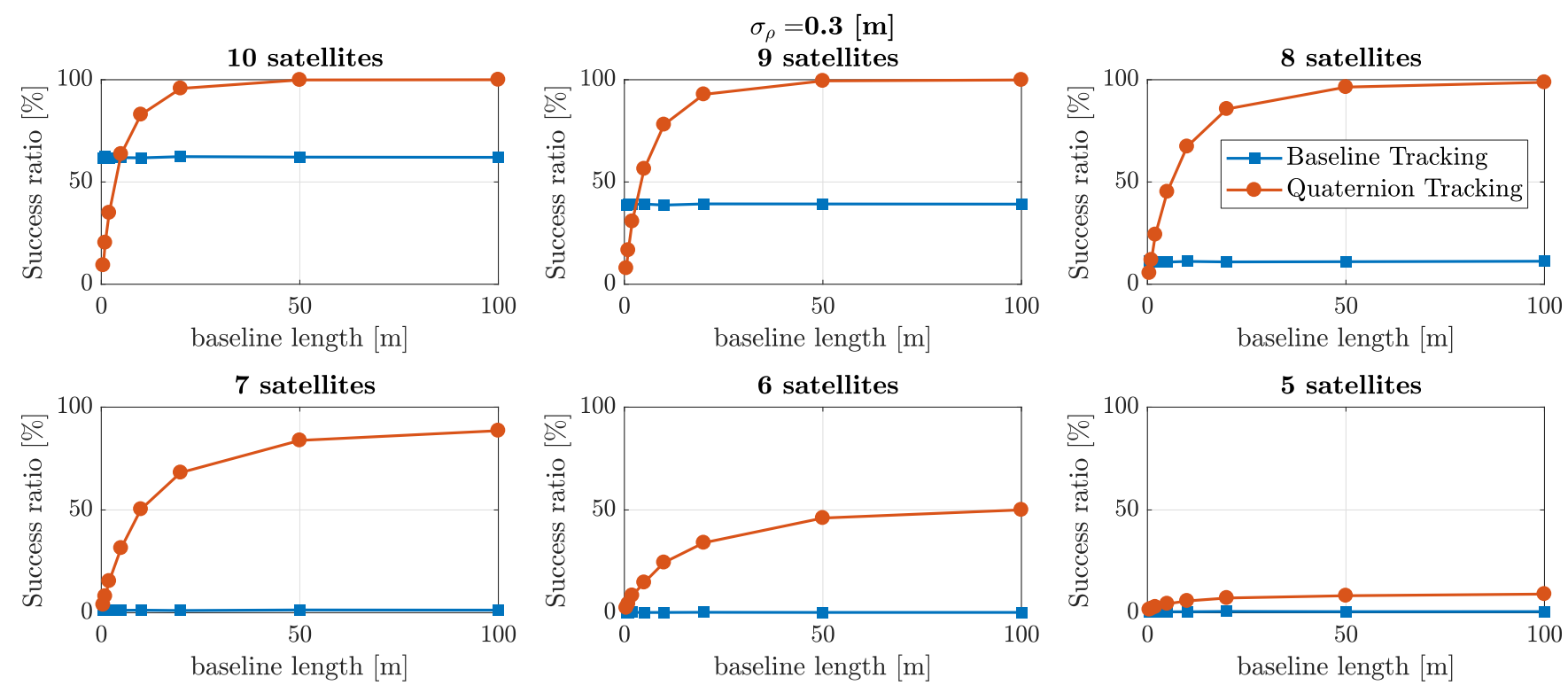

Figure 4: Success ratio against baseline length for $n=\{10,9,8,7,6,5\}$ satellites in view, given a deviation of the code noise of $\sigma_{\rho}=30[\mathrm{~cm}]$, for both the classical LAMBDA tracking the baselines in the ECEF frame and the proposed quaternion-based attitude determination problem (in a snapshot manner).

\section{IMU/GNSS Attitude Determination Performance}

This simulation scenario aims at addressing the performance of the multisensor recursive attitude, based on the ESKF for the estimation of the float solution and employing the quaternion GNSS attitude model as observation model. The number of satellites tracked is derived based on actual data recorded during an actual measurement campaign [26], with often drops in the number of satellites tracked due to the challenging environment. Satellite observability and the configuration of the antennas and the IMU on the body frame of the vehicle is as illustrated in Fig. 5. The experiment consists of a Monte Carlo simulation of $10^{3}$ samples . For the simulation of the code and phase observations, random normal noise is added, following the elevation-based stochastic model from Eq. (25), with $a, b=4 \mathrm{~mm}$.

The angular rate observations are derived from the simulated rotation over time. For the initial rotation simulation, the roll, pitch and yaw are represented as sinusoidal functions of different frequencies and amplitudes, replicating the original dynamics of the vessel (for instance, the pitch is influenced by the small waves of the river, creating a sinusoidal function of a frequency of around one second of a couple of degrees of amplitude). The noise characteristics of the simulated gyroscope are done according to the IMU model actually used for the actual measurement campaign, the Sensonor STIM300 [38]. The kinematics of the gyroscope biases is modelled as random walk. In Fig. 6, the bias estimation over time for the ESKF and the fixed solutions is shown. Only after approximately twenty seconds, the first fix solutions are reached, managing to converge to the right gyroscope biases from that moment. Such fast convergence to the right gyroscope biases is only possible due to the fast rate attitude change along the three rotation axis. On a real scenario, this estimation becomes far more challenging, due to the small misalignment between the IMU and the vehicle local frame axes, as well as the steady change in the roll and pitch (e.g., land vehicles barely experiment any change on their roll and pitch).

Table 3 shows the attitude performance, in terms of success rate, for classical baseline tracking against the proposed ESKF for a variety for multi-GNSS, multi-frequency scenarios. In general, AD improves with the addition of a second frequency rather than with a second constellation. In general, baseline tracking provides a much poorer 

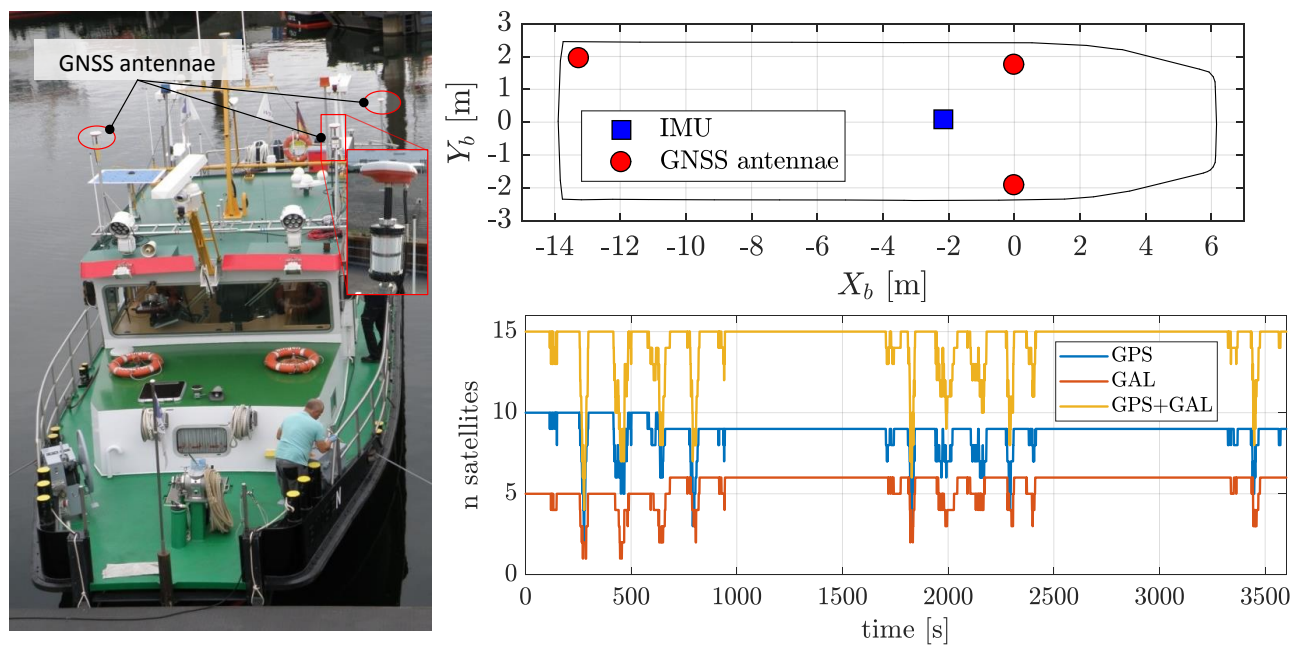

Figure 5: On the left, vessel whose antenna distribution is used for the simulation of the observations. On the top right, spatial distribution of the sensors within the body frame. On the bottom right, the number of tracked satellites over GPS and Galileo constellations on $L 1$ and $E 1$ frequencies.

performance than ESKF, whose success ratio is only relatively high for the case that GPS and Galileo are used along multiple frequencies. Except for the short convergence time in the estimation of the inertial biases, ESKF successfully estimates the integer ambiguities for the complete duration of the scenario.

Table 3: Ambiguity success rate for attitude estimation based on classical baseline tracking and the proposed ESKF, for multiple GNSS cases.

\begin{tabular}{lcc}
\hline & Baseline Tracking [\%] & ESKF [\%] \\
\hline GPS L1 & 0.08 & 97.11 \\
GPS L1+GAL E1 & 13.17 & 98.01 \\
GPS L1,L2 & 71.14 & 99.58 \\
GPS L1,L2+GAL E1,E5a & 94.03 & 99.89 \\
\hline
\end{tabular}

\section{CONCLUSION}

This paper presents a comprehensive description for applying the quaternion rotation parametrization to the GNSSbased attitude determination problem. The recursive estimation of the attitude and the additional fusion of angular rate observations motivate the proposal of an Error State Kalman Filter (ESKF), which bypasses the major challenges related to attitude filtering: on the one side, the gimbal lock singularity is completely avoided, while the attitude state representation remains minimal and away from possible numerical instabilities when estimating the covariance of the system. While extensive research has been carried out with regards to ambiguity resolution and attitude determination, especially within the work series of Teunissen and Giorgi [12, 39-41], recursive estimation of GNSS carrier phase-based attitude has been not fully exploited within the navigation community. This paper is intended to serve as basic guide for the implementation of ESKF for the GNSS+IMU attitude finding. The performance of the proposed method has been assessed in a twofold manner: i) a static and memoryless GNSS scenario is simulated via a Monte Carlo experimentation via different baseline lengths, number of observations and code noise. This experiment compares the performance of constraint float finding, in form of quaternion rotation estimation, against classical baseline tracking in the global frame. It becomes evident that, given that the quaternion rotation preserves the baseline length and relative orientation among baselines, its float estimation would be better than its state-of-art adversary. 


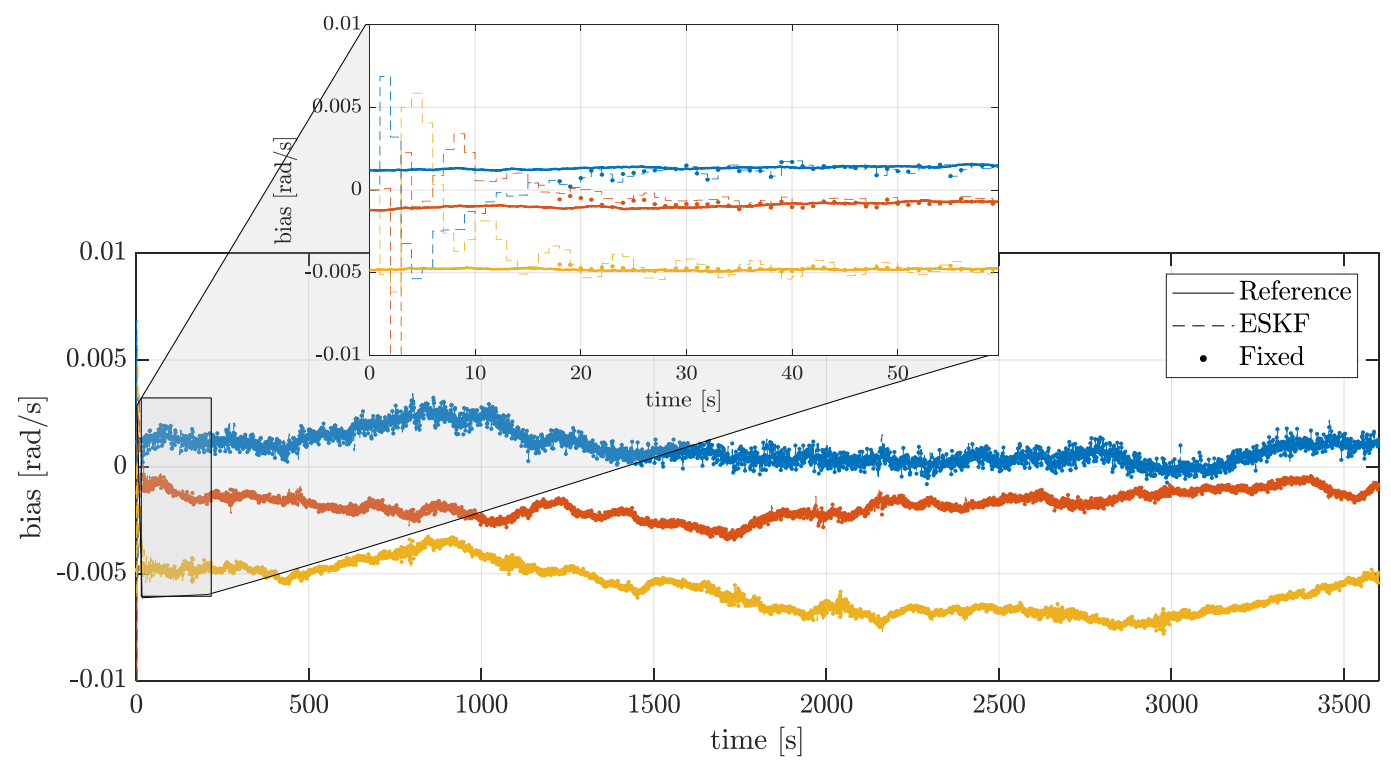

Figure 6: Estimation of the gyroscope biases over time for a particular Monte Carlo simulation (GPS L1+L2). Around twenty seconds after the movement is started, the filter converges to the right estimation for these and a fix attitude solution is also found.

\section{ACKNOWLEDGMENT}

Daniel Medina would like to thank Gabriele Giorgi for his fruitful comments and review on the text.

\section{APPENDIX I: JACOBIAN WITH RESPECT TO THE QUATERNION}

Given a rotation of the vector $\boldsymbol{v}$ with the quaternion rotation $\boldsymbol{q}$, the Jacobian $\boldsymbol{J}_{q}(\boldsymbol{v})$ of such operation with respect to the quaternion is given as follows:

$$
\boldsymbol{J}_{q}(\boldsymbol{v})=\frac{\partial\left(\boldsymbol{q} \circ \boldsymbol{v} \circ \boldsymbol{q}^{*}\right)}{\partial \boldsymbol{q}}
$$

For convenience, we will use a simplified notation for the quaternion, $\boldsymbol{q}=\left[q_{0}, \boldsymbol{q}_{\boldsymbol{u}}^{\top}\right]^{\top}$. A short reminder on quaternion multiplication:

$$
\boldsymbol{p} \circ \boldsymbol{q}=\left[\begin{array}{c}
p_{0} q_{0}-\boldsymbol{p}_{\boldsymbol{u}}^{\top} \boldsymbol{q}_{\boldsymbol{u}} \\
p_{0} \boldsymbol{q}_{\boldsymbol{u}}+q_{0} \boldsymbol{p}_{\boldsymbol{u}}+\boldsymbol{p}_{\boldsymbol{u}} \times \boldsymbol{q}_{\boldsymbol{u}}
\end{array}\right]
$$

Proceeding with the Jacobian derivation, we can expand the rotation operator as:

$$
\begin{aligned}
\boldsymbol{q} \circ \boldsymbol{v} \circ \boldsymbol{q}^{*} & =\left[\begin{array}{l}
q_{0} \\
\boldsymbol{q}_{\boldsymbol{u}}
\end{array}\right] \circ\left[\begin{array}{l}
0 \\
\boldsymbol{v}
\end{array}\right] \circ\left[\begin{array}{c}
q_{0} \\
-\boldsymbol{q}_{\boldsymbol{u}}
\end{array}\right] \\
& =\left[\begin{array}{c}
-\boldsymbol{q}_{\boldsymbol{u}}^{\top} \boldsymbol{v} \\
q_{0} \boldsymbol{v}+\boldsymbol{q}_{\boldsymbol{u}} \times \boldsymbol{v}
\end{array}\right] \circ\left[\begin{array}{c}
q_{0} \\
-\boldsymbol{q}_{\boldsymbol{u}}
\end{array}\right]
\end{aligned}
$$

from here, let us pay attention solely to the imaginary part:

$$
\begin{aligned}
\boldsymbol{q} \circ \boldsymbol{v} \circ \boldsymbol{q}^{*} & =-\boldsymbol{q}_{\boldsymbol{u}}^{\top} \boldsymbol{v}\left(-\boldsymbol{q}_{\boldsymbol{u}}\right)+q_{0}\left(q_{0} \boldsymbol{v}+\boldsymbol{q}_{\boldsymbol{u}} \times \boldsymbol{v}\right)+\left(q_{0} \boldsymbol{v}+\boldsymbol{q}_{\boldsymbol{u}} \times \boldsymbol{v}\right) \times\left(-\boldsymbol{q}_{\boldsymbol{u}}\right) \\
& =\boldsymbol{q}_{\boldsymbol{u}}^{\top} \boldsymbol{v} \boldsymbol{q}_{\boldsymbol{u}}+q_{0}^{2} \boldsymbol{v}+q_{0} \boldsymbol{q}_{\boldsymbol{u}} \times \boldsymbol{v}-q_{0} \boldsymbol{v} \times \boldsymbol{q}_{\boldsymbol{u}}+\left(\boldsymbol{q}_{\boldsymbol{u}} \times \boldsymbol{v}\right) \times\left(-\boldsymbol{q}_{\boldsymbol{u}}\right)
\end{aligned}
$$

Considering the vectorial product properties:

$$
\begin{gathered}
a \times b=-b \times a \\
a \times(b \times c)=\left(a^{\top} c\right) b-\left(a^{\top} b\right) c
\end{gathered}
$$


we can group $q_{0} \boldsymbol{q}_{\boldsymbol{u}} \times \boldsymbol{v}-q_{0} \boldsymbol{v} \times \boldsymbol{q}_{\boldsymbol{u}}=-2 q_{0} \boldsymbol{v} \times \boldsymbol{q}_{\boldsymbol{u}}$. Substituting the vectorial product for the skew operator $[\bullet \times]$, to facilitate the matrix formulation, and applying Eq. 60, we can reformulate the quaternion operator as:

$$
\boldsymbol{q} \circ \boldsymbol{v} \circ \boldsymbol{q}^{*}=q_{0}^{2} \boldsymbol{v}-2 q_{0}[\boldsymbol{v} \times] \boldsymbol{q}_{\boldsymbol{u}}+2\left(\boldsymbol{q}_{\boldsymbol{u}}^{\top} \boldsymbol{v}\right) \boldsymbol{q}_{\boldsymbol{u}}-\left(\boldsymbol{q}_{\boldsymbol{u}}^{\top} \boldsymbol{q}_{\boldsymbol{u}}\right) \boldsymbol{v}
$$

From here, deriving the partial derivatives of the quaternion results uncomplicated:

$$
\begin{gathered}
\frac{\partial\left(\boldsymbol{q} \circ \boldsymbol{v} \circ \boldsymbol{q}^{*}\right)}{\partial q_{0}}=2 q_{0} \boldsymbol{v}-2[\boldsymbol{v} \times] \boldsymbol{q}_{\boldsymbol{u}} \\
\frac{\partial\left(\boldsymbol{q} \circ \boldsymbol{v} \circ \boldsymbol{q}^{*}\right)}{\partial \boldsymbol{q}_{\boldsymbol{u}}}=-2 q_{0}[\boldsymbol{v} \times]+2\left(\boldsymbol{q}_{\boldsymbol{u}}^{\top} \boldsymbol{v} I_{3}+\boldsymbol{q}_{\boldsymbol{u}} \boldsymbol{v}^{\top}\right)-2 \boldsymbol{v} \boldsymbol{q}_{\boldsymbol{u}}^{\top}
\end{gathered}
$$

obtaining finally

$$
\boldsymbol{J}_{q}(\boldsymbol{v})=2\left[q_{0} \boldsymbol{v}-[\boldsymbol{v} \times] \boldsymbol{q}_{\boldsymbol{u}},-q_{0}[\boldsymbol{v} \times]+\boldsymbol{q}_{\boldsymbol{u}}^{\top} \boldsymbol{v} \boldsymbol{I}_{3}+\boldsymbol{q}_{\boldsymbol{u}} \boldsymbol{v}^{\top}-\boldsymbol{v} \boldsymbol{q}_{\boldsymbol{u}}^{\top}\right], \quad \boldsymbol{J}_{q}(\boldsymbol{v}) \in \mathbb{R}^{3 \times 4}
$$

\section{REFERENCES}

[1] Peter Teunissen and Oliver Montenbruck. Springer handbook of global navigation satellite systems. Springer, 2017.

[2] Peter Teunissen. The lambda method for the GNSS compass. Artificial Satellites, 41(3):89-103, 2006.

[3] Peter JG Teunissen. Least-squares estimation of the integer GPS ambiguities. In Invited lecture, section IV theory and methodology, IAG general meeting, Beijing, China, 1993.

[4] Paul De Jonge, Christian Tiberius, et al. The LAMBDA Methods for Integer Ambiguity Estimation: Implementation Aspects, volume 12. Verlag der Delft Univers. of Technolog., 1996.

[5] Peter Joosten and Christian Tiberius. Lambda: Faqs. GPS Solutions, 6(1-2):109-114, 2002.

[6] André Hauschild, Georg Grillmayer, Oliver Montenbruck, Markus Markgraf, and Peter Vörsmann. Gps based attitude determination for the flying laptop satellite. In Small satellites for earth observation, pages 211-220. Springer, 2008.

[7] Leen Vander Kuylen, Frank Boon, and Andrew Simsky. Attitude determination methods used in the polarx2@ multi-antenna GPS receiver. Proceedings of ION-GPS 2005, 2005.

[8] L Vander Kuylen, P Nemry, F Boon, A Simsky, and Septentrio NV. Comparison of attitude performance for multi-antenna receivers. European Journal of Navigation, 4(2):1-9, 2006.

[9] Hui-nan Wang and Jin-dong Ying. LAMBDA method for rigid body attitude determination based on GPS. Acta Aeronautica et Asronautica Sinica, 22(1):61-63, 2001.

[10] Gabriele Giorgi. GNSS carrier phase-based attitude determination: estimation and applications. 2011.

[11] PJG Teunissen and G Giorgi. To what extent can standard GNSS ambiguity resolution methods be used for single-frequency epoch-by-epoch attitude determination? Proc. of the 22nd ION GNSS, pages 235-242, 2009.

[12] Peter JG Teunissen, Gabriele Giorgi, and Peter J Buist. Testing of a new single-frequency GNSS carrier phase attitude determination method: land, ship and aircraft experiments. GPS solutions, 15(1):15-28, 2011.

[13] Nandakumaran Nadarajah, Peter JG Teunissen, Peter J Buist, and Peter Steigenberger. First results of instantaneous GPS/Galileo/COMPASS attitude determination. In 2012 6th ESA Workshop on Satellite Navigation Technologies (Navitec 2012) 8 European Workshop on GNSS Signals and Signal Processing, pages 1-8. IEEE, 2012.

[14] Gabriele Giorgi and Peter JG Teunissen. Carrier phase GNSS attitude determination with the multivariate constrained LAMBDA method. In 2010 IEEE Aerospace Conference, pages 1-12. IEEE, 2010.

[15] Gabriele Giorgi and Peter Teunissen. GNSS carrier phase-based attitude determination. In Recent advances in aircraft technology, pages 193-220. InTech, 2012.

[16] G Giorgi, PJG Teunissen, S Verhagen, and PJ Buist. Improving the GNSS attitude ambiguity success rate with the multivariate constrained lambda method. In Geodesy for Planet Earth, pages 941-948. Springer, 2012.

[17] Gabriele Giorgi and Peter JG Teunissen. Multivariate GNSS Attitude Integrity: The Role of Affine Constraints. In VIII HotineMarussi Symposium on Mathematical Geodesy, pages 309-315. Springer, 2015.

[18] Safoora Zaminpardaz, Peter Teunissen, and Nandakumaran Nadarajah. IRNSS/NavIC L5 attitude determination. Sensors, 17(2):274, 2017.

[19] Paul D Groves. Principles of GNSS, inertial, and multisensor integrated navigation systems. Artech house, 2013.

[20] Daniel Arias Medina, Michailas Romanovas, Iván Herrera-Pinzón, and Ralf Ziebold. Robust position and velocity estimation methods in integrated navigation systems for inland water applications. In 2016 IEEE/ION Position, Location and Navigation Symposium (PLANS), pages 491-501. IEEE, 2016.

[21] Christian Eling, Philipp Zeimetz, and Heiner Kuhlmann. Development of an instantaneous GNSS/MEMS attitude determination system. GPS solutions, 17(1):129-138, 2013. 
[22] Omar García Crespillo, Oliver Heirich, and Andreas Lehner. Bayesian GNSS/IMU tight integration for precise railway navigation on track map. In 2014 IEEE/ION Position, Location and Navigation Symposium-PLANS 2014, pages 999-1007. IEEE, 2014.

[23] Stergios I Roumeliotis, Gaurav S Sukhatme, and George A Bekey. Circumventing dynamic modeling: Evaluation of the error-state Kalman filter applied to mobile robot localization. In Proceedings 1999 IEEE International Conference on Robotics and Automation (Cat. No. 99CH36288C), volume 2, pages 1656-1663. IEEE, 1999.

[24] F Landis Markley. Attitude error representations for Kalman filtering. Journal of guidance, control, and dynamics, 26(2):311-317, 2003.

[25] Nikolas Trawny and Stergios I Roumeliotis. Indirect Kalman filter for 3D attitude estimation. University of Minnesota, Dept. of Comp. Sci. \& Eng., Tech. Rep, 2:2005, 2005.

[26] Daniel Medina, Anja Heßelbarth, Rauno Büscher, Ralf Ziebold, and Jesús García. On the Kalman Filtering Formulation for RTK Joint Positioning and Attitude Quaternion Determination. In 2018 IEEE/ION Position, Location and Navigation Symposium (PLANS), pages 597-604. IEEE, 2018.

[27] John Stillwell. Naive lie theory. Springer Science \& Business Media, 2008.

[28] William Rowan Hamilton. Elements of quaternions. Longmans, Green, \& Company, 1866.

[29] Annett Chilian, Heiko Hirschmüller, and Martin Görner. Multisensor data fusion for robust pose estimation of a six-legged walking robot. In 2011 IEEE/RSJ International Conference on Intelligent Robots and Systems, pages 2497-2504. IEEE, 2011.

[30] James Maley and Guoquan Huang. Unit quaternion-based parameterization for point features in visual navigation. In 2018 IEEE/RSJ International Conference on Intelligent Robots and Systems (IROS), pages 6880-6886. IEEE, 2018.

[31] Ern J Lefferts, F Landis Markley, and Malcolm D Shuster. Kalman filtering for spacecraft attitude estimation. Journal of Guidance, Control, and Dynamics, 5(5):417-429, 1982.

[32] Joan Sola. Quaternion kinematics for the error-state Kalman filter. arXiv preprint arXiv:1711.02508, 2017.

[33] Christian Eling, Lasse Klingbeil, and Heiner Kuhlmann. Development of an RTK-GPS system for precise real-time positioning of lightweight UAVs. In Proc. of, 2014.

[34] Peter JG Teunissen. An optimality property of the integer least-squares estimator. Journal of geodesy, 73(11):587-593, 1999.

[35] John L Crassidis, F Landis Markley, and Yang Cheng. Survey of nonlinear attitude estimation methods. Journal of guidance, control, and dynamics, 30(1):12-28, 2007.

[36] Shu Ting Goh and Kay-Soon Low. Survey of Global-Positioning-System-based attitude determination algorithms. Journal of Guidance, Control, and Dynamics, 40(6):1321-1335, 2017.

[37] Venkatesh Madyastha, Vishal Ravindra, Srinath Mallikarjunan, and Anup Goyal. Extended Kalman filter vs. error state Kalman filter for aircraft attitude estimation. In AIAA Guidance, Navigation, and Control Conference, page 6615, 2011.

[38] Sensonor. Sensonor STIM300 inertial measurement unit datasheet, 2013. [Online; accessed 25-September-2019].

[39] Peter Teunissen. A general multivariate formulation of the multi-antenna GNSS attitude determination problem. Artificial Satellites, $42(2): 97-111,2007$.

[40] G Giorgi, PJG Teunissen, and PJ Buist. A search and shrink approach for the baseline constrained LAMBDA method: Experimental results. In Proceedings International Symposium GPS/GNSS. Curran Associates, 2008.

[41] Gabriele Giorgi, Peter JG Teunissen, and Tim P Gourlay. Instantaneous global navigation satellite system (GNSS)-based attitude determination for maritime applications. IEEE Journal of oceanic engineering, 37(3):348-362, 2012. 